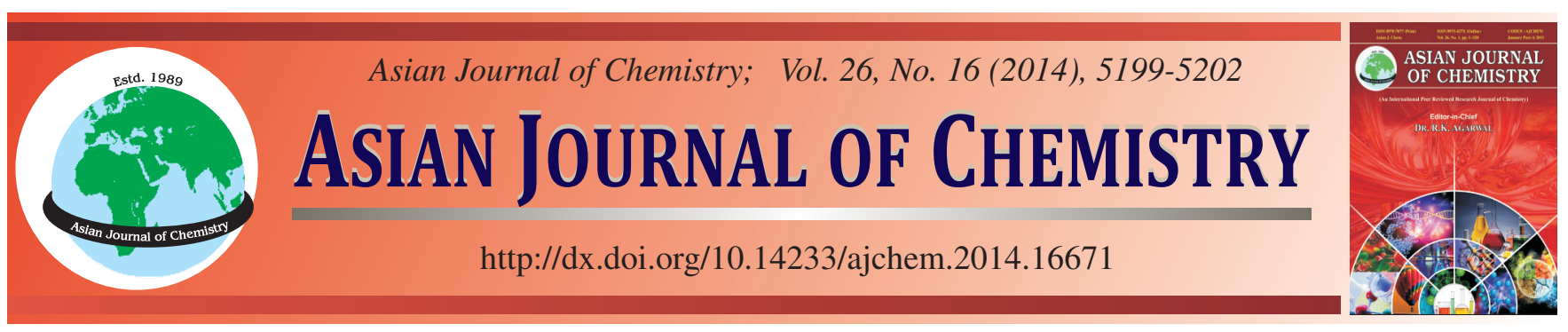

\title{
Resonance Rayleigh Scattering Spectra of CdTe Quantum Dots-Dermatan Sulfate System and its Analytical Applications
}

\author{
DAN LiU ${ }^{1,2, *}$
}

${ }^{1}$ Sichuan University of Arts and Science, Dazhou 635000, P.R. China

${ }^{2}$ School of Chemistry and Chemical Engineering; Southwest University, Chongqing 400715, P.R. China

*Corresponding author: E-mail: dandanjacky@sina.com; 330548103@qq.com

Received: 11 November 2013

Accepted: 13 March 2014;

Published online: 28 July 2014;

AJC-15661

\begin{abstract}
In this paper, CdTe quantum dots with the diameter of 2-3 nm were synthesized in aqueous solution with cysteamine as stabilizing agent. The interaction between cysteamine-CdTe quantum dots and dermatan sulfate was studied by resonance Rayleigh scattering spectra. It showed that the intensities of resonance Rayleigh scattering of cysteamine-CdTe quantum dots -dermatan sulfate were enhanced greatly, the resonance Rayleigh scattering intensities $\left(\Delta I_{R R S}\right)$ was directly proportional to the concentration of dermatan sulfate. The linear ranges and the detection limits were $0.0039-0.6$ and $1.2 \mathrm{ng} \mathrm{mL}^{-1}$. The proposed methods exhibited higher sensitivity, so that they are more suitable for the determination of trace amounts dermatan sulfate. The optimum conditions, the influencing factors and the effects of coexisting substances on the reaction were investigated. The result indicated that the method can be applied to the determination of dermatan sulfate in serum and urine samples with satisfactory results. The reasons of resonance Rayleigh scattering enhancement were also discussed.
\end{abstract}

Keywords: Cysteamine capped CdTe quantum dots, CdTe quantum dots, Resonance Rayleigh scattering, Dermatan sulfate.

\section{INTRODUCTION}

Dermatan sulfate, a sulfated glycosaminoglycan, is composed of repeated two sugar units which are made up of 2-acetyl-4-sulfuric acid-D-galactose and L-iduronic acid ${ }^{1}$.

It has been reported that dermatan sulfate can be used to anticoagulant, antithrombotic, anti-inflammatory, antiviral, antiproliferative and protection of vascular wall. The bleeding side effect caused by dermatan sulfate is small. It can prevent the formation of thrombus and thrombolytic effect. Therefore, it is a kind of promising new anti-thrombus medicine and plays an important role in modern medicinal field ${ }^{2}$. However, there are only a few reports about the determination of dermatan sulfate . It is significant for studying the determination of dermatan sulfate .

Some methods have been developed for the determination of dermatan sulfate, including high performance liquid chromatography (HPLC) ${ }^{3}$, electrophoresis ${ }^{4}$, spectrophotometry $^{5}$, resonance Rayleigh scattering ${ }^{6}$, etc. Resonance Rayleigh scattering (RRS) has drawn much more attention due to its high sensitivity and simplicity ${ }^{7}$. It has been reported that the resonance Rayleigh scattering technique can be used for the determination of dermatan sulfate. However, use of the enhancement of resonance Rayleigh scattering intensity and quantum dots as probes to determine dermatan sulfate has not been reported. In this paper, the interaction between cysteamine-CdTe quantum dots and dermatan sulfate was investigated by resonance Rayleigh scattering. In acetic acidsodium acetate buffer solution $(\mathrm{pH}=4.8)$, cysteamine-CdTe quantum dots reacted with dermatan sulfate and the products resulted in a great enhancement of resonance Rayleigh scattering and non-linear scattering such as second-order scattering (SOS) and frequency doubling scattering (FDS). The increments of scattering intensity $(\Delta \mathrm{I})$ were directly proportional to the concentrations of dermatan sulfate in the range of 0.0039-0.6 $\mu \mathrm{g} \mathrm{mL}^{-1}$. The proposed method was highly sensitive, good selective, simple, rapid and low detection limits. The method can be applied to the determination of dermatan sulfate in serum and urine samples with satisfactory results.

\section{EXPERIMENTAL}

Cysteamine was purchased from Tianjin institute of fine chemicals retrocession (Tianjin, China), Te was obtained from Shanghai Meixing Chemical Reagent Co. (Shanghai, China). $\mathrm{CdCl}_{2} \cdot 2.5 \mathrm{H}_{2} \mathrm{O}$ was purchased from Shanghai Chemicals Reagent Co. (Shanghai, China), $\mathrm{NaBH}_{4}$ was obtained from Tianjin Huanwei Fine Chemical Co., (Tianjin, China), $\mathrm{H}_{2} \mathrm{SO}_{4}$ and $\mathrm{NaOH}$ were obtained from Chongqing Chuandong Chemicals Reagent Co. (Chongqing, China). $1 \times 10^{-4} \mathrm{mg} \mathrm{mL}^{-1}$ stock solution of dermatan sulfate (DS) was prepared by dissolving solid $0.0101 \mathrm{~g}$ 
dermatan sulfate (Sigma) in water and diluting to the mark. The working solution was prepared by diluting the stock solution to $1 \times 10^{-5} \mathrm{mg} \mathrm{mL}^{-1}$. $\mathrm{CH}_{3} \mathrm{COONa}-\mathrm{CH}_{3} \mathrm{COOH}$ buffer solutions with different $\mathrm{pH}$, which were adjusted by $\mathrm{pH}$ meter, were prepared by mixing $\mathrm{CH}_{3} \mathrm{COOH}$ and $\mathrm{CH}_{3} \mathrm{COONa}$ and used to control the acidity of the aqueous medium.

All the reagents used were of analytical reagent (A.R.) grade and doubly distilled water was used throughout. All of the experiments were performed at the room temperature.

A Hitachi F-2500 spectroflurophotometer (Tokyo, Japan) was used for measuring the scattering intensities with the slits (EX/EM) of $5 \mathrm{~nm} / 5 \mathrm{~nm}$. A UV-8500 spectrophotometer (Tianmei Co., Shanghai, China) was used for recording the absorption spectra. TECNAI-10 transmission electron microscopy (TEM) (Philips Company, Holland) was used to observe the appearance and the size of nanoparticles. A PHS-3C pH meter (Shanghai Analytical Instrument Factory, China) was used for measuring $\mathrm{pH}$.

Aqueous colloids of CdTe quantum dots solution were prepared according to published methods ${ }^{8} .0 .0752 \mathrm{~g} \mathrm{CdCl}_{2} \cdot 2.5 \mathrm{H}_{2} \mathrm{O}$, $0.01329 \mathrm{~g}$ cysteamine and $15 \mathrm{~mL}$ of deionized water were placed in $250 \mathrm{~mL}$ three-necked flask, followed by adjusting the $\mathrm{pH}$ to 5-6 by dropwise addition of $1 \mathrm{~mol} / \mathrm{L} \mathrm{NaOH}$ solution.

Under $\mathrm{N}_{2}$ atmosphere, $0.0151 \mathrm{~g}$ Te and an appropriate amount deionized water were added to a $50 \mathrm{~mL}$ three-necked flask which was connected to a $250 \mathrm{~mL}$ three-necked flask by earway. Under $\mathrm{N}_{2}$ atmosphere for $20 \mathrm{~min}$, excessive sodium borohydride was added under magnetic stirring at room temperature. After Te was reacted completed, $0.5 \mathrm{~mol} / \mathrm{L} \mathrm{H}_{2} \mathrm{SO}_{4}$ was added dropwise until the reaction was completed. At this time, the solution in $250 \mathrm{~mL}$ three-necked flask turned orange. Then the resulting mixture was subjected to reflux at $369 \mathrm{~K}$ for $2 \mathrm{~h}$. At last, Orange-red quantum dots were obtained. (the concentration of $\mathrm{Cd}^{2+}$ was $2.5 \times 10^{-3} \mathrm{~mol} / \mathrm{L}$ )

$0.6 \mathrm{~mL}$ CdTe quantum dots, $1 \mathrm{~mL} \mathrm{CH}_{3} \mathrm{COONa}-\mathrm{CH}_{3} \mathrm{COOH}$ buffer solution $(\mathrm{pH}=4.8)$ and an appropriate amount of dermatan sulfate were added into a $10 \mathrm{~mL}$ volumetric flask, then diluted with deionized water to the mark and mixed thoroughly with gentle shake. After incubation for $10 \mathrm{~min}$, the resonance Rayleigh scattering spectra of solution were examined. The resonance Rayleigh scattering intensity $\left(\Delta \mathrm{I}_{\mathrm{RRS}}\right)$ of CdTe quantum dots-dermatan sulfate system was measured as following formulas: $\Delta \mathrm{I}_{\mathrm{RRS}}=\mathrm{I}_{\mathrm{RRS}} \mathrm{I}^{0}{ }_{\mathrm{RRS}}$; $\mathrm{I}_{\mathrm{RRS}}$ and $\mathrm{I}_{\mathrm{RRS}}^{0}$ were the scattering intensities of CdTe quantum dots-dermatan sulfate system and the CdTe quantum dots blank, respectively.

\section{RESULTS AND DISCUSSION}

TEM images: The shape and diameter of the CdTe quantum dots were observed by TEM. The TEM image shows clearly that these quantum dots are monodisperse. The cysteamine capped CdTe quantum dots were successfully synthesized according to the protocol described in Section 2.3. The average size of the particle was about 2-3 nm analyzed by Fig. 1.

Resonance Rayleigh scattering spectra: Fig. 2 shown the resonance Rayleigh scattering spectra of CdTe quantum dots-dermatan sulfate system. It was found that the resonance Rayleigh scattering intensity of CdTe quantum dots and dermatan sulfate were very weak. When dermatan sulfate reacted with CdTe quantum dots solution to form a complex, the resonance

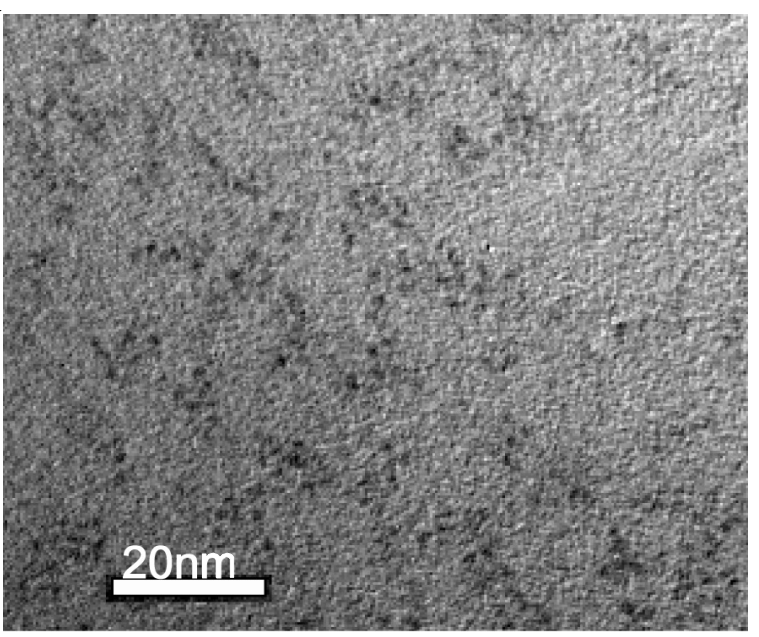

Fig. 1. TEM images of CdTe quantum dots

Rayleigh scattering intensity of the solution can be greatly enhanced and the enhancement of resonance Rayleigh scattering intensity was directly proportional to the concentration of dermatan sulfate in the range of $0.2-0.6 \mu \mathrm{g} \mathrm{mL}-1$. The maximum scattering peak position of CdTe quantum dotsdermatan sulfate is located at $343 \mathrm{~nm}$.

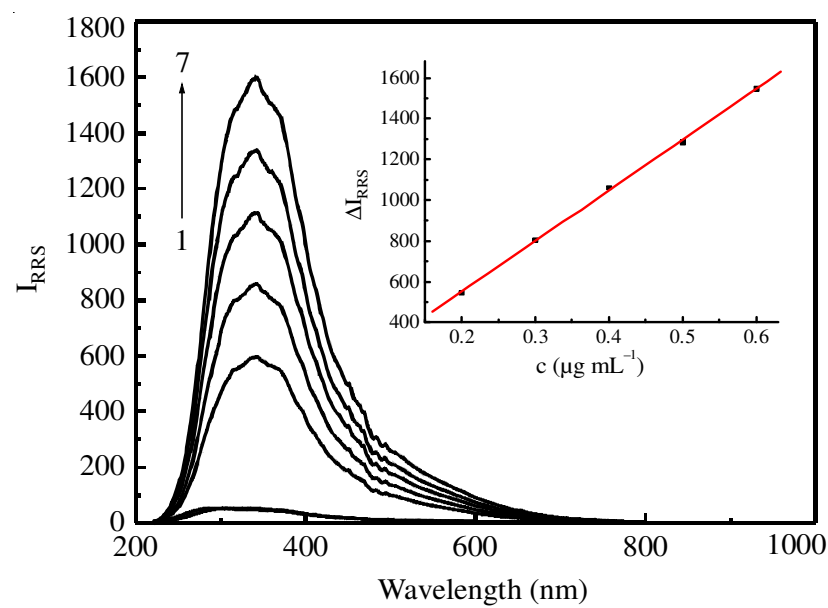

Fig. 2. Resonance Rayleigh scattering spectra of the CdTe quantum dotsdermatan sulfate system. 1. CdTe-quantum dots: $c(\mathrm{CdTe}$-quantum dots $)=2.5 \times 10^{-4} \mathrm{~mol} \mathrm{~L}^{-1} ; 2$. dermatan sulfate: $\mathrm{c}($ dermatan sulfate $)$ $=1 \mu \mathrm{g} \mathrm{mL}^{-1}$; 3-7. CdTe-quantum dots -dermatan sulfate: c(dermatan sulfate): $0.2,0.3,0.4,0.5,0.6 \mu \mathrm{g} \mathrm{mL}$

Effect of the acidity on resonance Rayleigh scattering: The influence of $\mathrm{pH}$ on the resonance Rayleigh scattering intensity of the CdTe QD-dermatan sulfate system was investigated. In this paper, $\mathrm{CH}_{3} \mathrm{COONa}-\mathrm{CH}_{3} \mathrm{COOH}$ buffer solution was selected to control the $\mathrm{pH}$ of analytical system. As Fig. 3 shown, the resonance Rayleigh scattering intensity of the CdTe QD-dermatan sulfate system was strongly affected by the $\mathrm{pH}$ value. The maximum of the resonance Rayleigh scattering intensity of the CdTe quantum dot-dermatan sulfate system was found at $\mathrm{pH} 4.8$. So, the buffer solution of $\mathrm{pH} 4.8$ was selected for further experiments. The optimum amount of $\mathrm{CH}_{3} \mathrm{COONa}-\mathrm{CH}_{3} \mathrm{COOH}$ buffer solution was $1 \mathrm{~mL}$.

Effect of the CdTe quantum dots concentration: The effect of the CdTe quantum dots concentration on the resonance Rayleigh scattering intensity of CdTe quantum dots-dermatan 


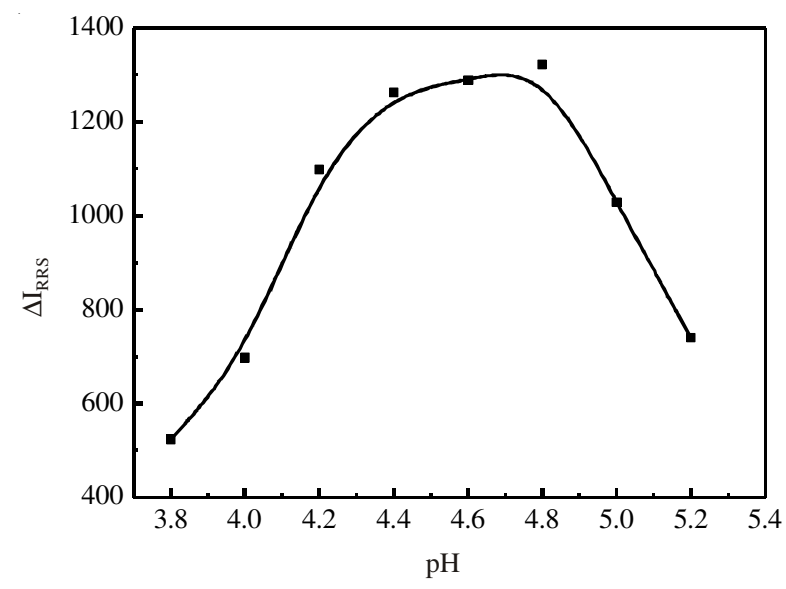

Fig. 3. Influence of $\mathrm{pH}$ to the resonance Rayleigh scattering intensity of the CdTe quantum dot-dermatan sulfate system; c(CdTe-quantum dots $)=2.5 \times 10^{-4} \mathrm{~mol} \mathrm{~L}^{-1} ; \mathrm{c}($ dermatan sulfate $)=1 \mu \mathrm{g} \mathrm{mL}$

sulfate system was studied and the result is presented in Fig. 4. It can be seen that the concentration of CdTe quantum dots had great influence on the resonance Rayleigh scattering intensity of CdTe quantum dots-dermatan sulfate system. When the concentration of CdTe quantum dots was low, the intensity of CdTe quantum dots-dermatan sulfate system was faint due to the incomplete reaction. With the increase in concentration of CdTe quantum dots, the resonance Rayleigh scattering intensity of CdTe quantum dots-dermatan sulfate system enhanced gradually. $0.4-1 \mathrm{~mL}$ of CdTe quantum dots was the optimum suitable amount and the concentration of CdTe quantum dots was $(1-2.5) \times 10^{-4} \mathrm{~mol} \mathrm{~L}^{-1}$, the relative intensities $(\Delta \mathrm{I})$ of CdTe quantum dots-dermatan sulfate system was stable and high. When the concentration of CdTe-quantum dots was too high, the resonance Rayleigh scattering intensity decreased. So, we selected $0.6 \mathrm{~mL}$ as the optimum amount of CdTe quantum dots.

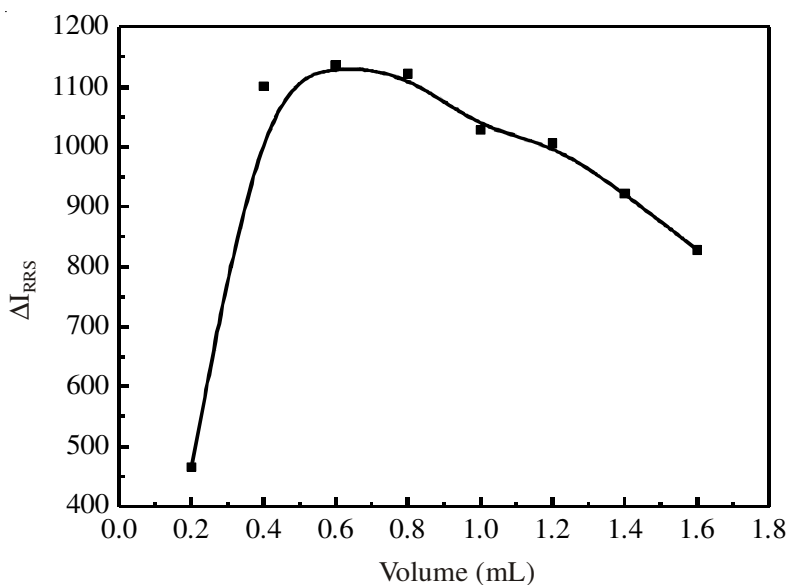

Fig. 4. Effect of the CdTe quantum dots concentration; c(CdTe quantum dots $)=2.5 \times 10^{-4} \mathrm{~mol} \mathrm{~L}^{-1} \mathrm{c}($ dermatan sulfate $)=1 \mu \mathrm{g} \mathrm{mL}{ }^{-1} ; \mathrm{pH}=4.8$

Effect of reaction time and the stability: The effect of reaction time on the resonance Rayleigh scattering intensity of CdTe quantum dots-dermatan sulfate system was also studied. According to the experimental results, it can be seen that the resonance Rayleigh scattering intensity achieved the maximum within $10 \mathrm{~min}$ and the resonance Rayleigh scattering intensity remained stable for $2 \mathrm{~h}$ when $\mathrm{CH}_{3} \mathrm{COONa}-\mathrm{CH}_{3} \mathrm{COOH}$ was buffer solution. Therefore, the experiment was performed after $10 \mathrm{~min}$

Reaction mechanism and the reasons for resonance Rayleigh scattering enhancement: The reasons for resonance Rayleigh scattering enhancement may be the following:

(1) As Fig. 5 shown, resonance Rayleigh scattering peak is located at its absorption band. When Rayleigh scattering wavelength was near to the absorption band, resonance Rayleigh scattering effect may be produced and leads to great enhancement of resonance Rayleigh scattering intensity.

(2) It is well known that the increase of the molecule volume directly effects the enhancement of scattering intensity ${ }^{9}$. In weak acidic buffer solution, dermatan sulfate is negatively charged while the surface of cysteamine-CdTe quantum dots is positively charged. It is easy for dermatan sulfate molecules to bind onto the surface of cysteamine-CdTe quantum dots via the electrostatic attraction and hydrophobic effect. With the increase of dermatan sulfate on the surface of cysteamineCdTe quantum dots, the surface of cysteamine-CdTe quantum dots become negatively charged which can be combined with another positively charged quantum dots by sulfate and results in the increase of molecular weight and volume. Therefore, the resonance Rayleigh scattering intensity of CdTe quantum dots and dermatan sulfate is very weak. When they combine to form binding products, the resonance Rayleigh scattering intensity of the system is greatly enhanced.

(3) After the stabilizing agents connect to the surface of quantum dots by $\mathrm{Cd}$-Te bond, quantum dots become positively charged compounds which contain many hydrophilic groups and can combine with water to form an aquo-complex. However, when CdTe quantum dots further bind with dermatan sulfate, their charges are neutralized, which causes the increase of the hydrophobicity of the binding products. In this case, the liquid-solid interface between the complex and water may form, which enhances the scattering intensities.

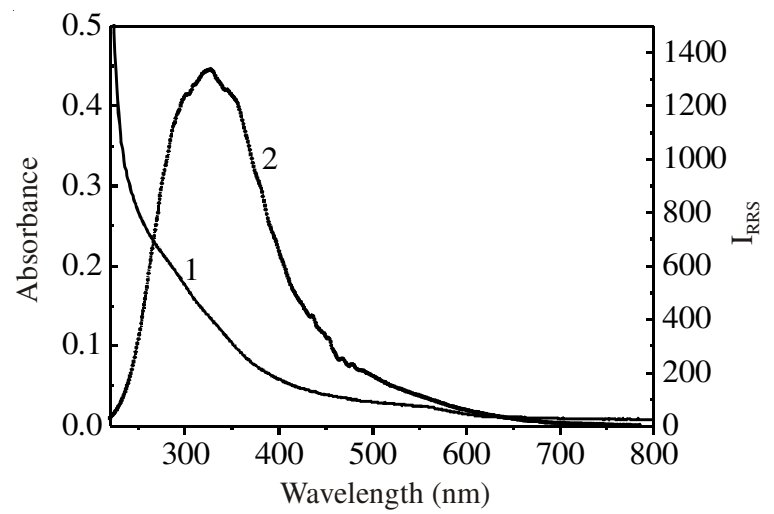

Fig. 5. Comparison between resonance Rayleigh scattering and absorption spectra; (1) Absorption spectrum; (2) resonance Rayleigh scattering spectrum; $\mathrm{c}($ dermatan sulfate $)=1 \mu \mathrm{g} \mathrm{mL}^{-1} ; \mathrm{c}(\mathrm{CdTe}$-quantum dots $)$ $=2.5 \times 10^{-4} \mathrm{mo} \mathrm{L}^{-1} ; \mathrm{pH}=4.8$

Calibration curve: Under the optimum conditions, the resonance Rayleigh scattering spectra of the binding products of CdTe quantum dots with different concentrations of dermatan sulfate solution were investigated. The enhancement of resonance Rayleigh scattering intensity was linear to the dermatan sulfate concentration in the range of $0.0039-0.6 \mu \mathrm{g} \mathrm{mL}^{-1}$ with the detection limit $(3 \sigma)$ of $1.2 \mathrm{ng} \mathrm{mL}^{-1}$ (Table-1). 


\begin{tabular}{|c|c|c|c|c|c|}
\hline \multicolumn{6}{|c|}{$\begin{array}{c}\text { TABLE-2 } \\
\text { EFFECTS OF FOREIGN SUBSTANCES (DERMATAN SULFATE: } 0.5 \mu \mathrm{g} \mathrm{mL}^{-1} \text { ) }\end{array}$} \\
\hline Foreign substance & $\begin{array}{l}\text { Concentration } \\
\left(\mu \mathrm{g} \mathrm{mL} \mathrm{mL}^{-1}\right)\end{array}$ & Relative error (\%) & Foreign substance & $\begin{array}{l}\text { Concentration } \\
\left(\mu \mathrm{g} \mathrm{mL}^{-1}\right)\end{array}$ & Relative error (\%) \\
\hline Lactose & 150.0 & 3.9 & $\mathrm{Cl}^{-}$ & 240.0 & -3.0 \\
\hline Glucose & 100.0 & -3.8 & $\mathrm{CH}_{3} \mathrm{COO}^{-}$ & 100.0 & 7.8 \\
\hline Sugar & 40.0 & 1.1 & $\mathrm{SO}_{4}{ }^{2-}$ & 12.0 & 1.8 \\
\hline Maltose & 100.0 & -3.5 & $\mathrm{CO}_{3}^{2-}$ & 200.0 & -3.8 \\
\hline Glycin & 60.0 & -2.0 & $\mathrm{NO}^{3-}$ & 80.0 & -5.5 \\
\hline L-cysteine & 300.0 & -4.8 & $\mathrm{NH}^{4+}$ & 50.0 & 2.3 \\
\hline L-glutamic acid & 100.0 & 2.5 & $\mathrm{Ba}^{2+}$ & 10.0 & -5.0 \\
\hline $\mathrm{HSA}^{\mathrm{a}}$ & 2.0 & 2.7 & $\mathrm{~K}^{+}$ & 80.0 & -5.5 \\
\hline $\mathrm{BSA}^{\mathrm{b}}$ & 50.0 & 3.8 & $\mathrm{Fe}^{3+}$ & 5.0 & 4.0 \\
\hline $\mathrm{VB}_{12}$ & 5.0 & -1.2 & $\mathrm{Mg}^{2+}$ & 12.0 & 5.8 \\
\hline Urea & 180.0 & -4.2 & $\mathrm{Ca}^{2+}$ & 120.0 & -3.0 \\
\hline Cyclodextrin & 100.0 & -3.6 & $\mathrm{Na}^{+}$ & 200.0 & -3.8 \\
\hline Citric acid & 40.0 & -4.6 & $\mathrm{~Pb}^{2+}$ & 50.0 & 1.7 \\
\hline
\end{tabular}

TABLE-3

\begin{tabular}{cccccc}
\multicolumn{5}{c}{ TABLE-3 } \\
\multicolumn{5}{c}{ DETERMINATION OF DS IN SERUM AND URINE SAMPLES } \\
\hline Sample & Total found $\left(\mu \mathrm{g} \mathrm{mL} \mathrm{mL}^{-1}, \mathrm{n}=5\right)$ & Added $\left(\mu \mathrm{g} \mathrm{m}^{-1}\right)$ & Total found $\left(\mu \mathrm{gL}^{-1}, \mathrm{n}=5\right)$ & Recovery $(\%, \mathrm{n}=5)$ & $\mathrm{RSD}(\%, \mathrm{n}=5)$ \\
\hline Serum 1 & ND & 0.6 & 0.589 & 98.2 & 1.5 \\
Serum 2 & ND & 0.3 & 0.292 & 97.4 & 97.5 \\
Urine 1 & ND & 0.6 & 0.585 & 95.8 & 1.7 \\
Urine 2 & ND & 0.3 & 0.287 & 95.8 & 5.7 \\
\hline
\end{tabular}

\begin{tabular}{ccccc}
\multicolumn{5}{c}{ TABLE-1 } \\
\hline ANALYTICAL PARAMETERS OF RRS METHOD \\
\hline Method & $\begin{array}{c}\text { Linear } \\
\text { equation } \\
\left(\mu \mathrm{g} \mathrm{m}^{-1}\right)\end{array}$ & $\mathrm{R}$ & $\begin{array}{c}\text { Detection } \\
\text { limit } \\
\left(\mathrm{ng} \mathrm{mL}^{-1}\right)\end{array}$ & $\begin{array}{c}\text { Linear } \\
\text { range } \\
\left(\mu \mathrm{gL}^{-1}\right)\end{array}$ \\
\hline RRS & $\Delta \mathrm{I}=2536.1 \mathrm{c}+46.31$ & 0.9997 & 1.2 & $0.0039-0.6$ \\
\hline
\end{tabular}

Effects of coexisting substances: Under optimum conditions, the influences of coexisting substances, such as amino acid, carbohydrate, protein and others, were tested in the presences of dermatan sulfate by resonance Rayleigh scattering method. The results were given in Table-2, it can be seen that the method has a good selectivity and can be used for the determination of dermatan sulfate in real samples.

Determination of dermatan sulfate in human serum albumin and urine samples: According to the experimental method, the amounts of dermatan sulfate in five fresh urine samples (healthy human) were tested. Fresh urine samples were treated with $1 \mathrm{~mL}$ acetonitrile and centrifuged. When the proteins in serum were separated thoroughly, $1 \mathrm{~mL}$ supernatants of the sample, $0.6 \mathrm{~mL}$ CdTe quantum dots and $1 \mathrm{~mL} \mathrm{CH}_{3} \mathrm{COONa}-$ $\mathrm{CH}_{3} \mathrm{COOH}$ buffer solution were added into $10 \mathrm{~mL}$ volumetric flask. The solution was diluted to the mark and mixed thoroughly. Every concentration was tested 5 times. The relative standard deviation (RSD) and recovery were tested by a standard addition method. The results were listed in Table-3.

\section{Conclusion}

In this paper, the interaction between CdTe quantum dots and dermatan sulfate was investigated by resonance Rayleigh scattering. In $\mathrm{CH}_{3} \mathrm{COONa}-\mathrm{CH}_{3} \mathrm{COOH}$ buffer solution, dermatan sulfate displayed negatively charged while the surface of cysteamine-CdTe quantum dots displayed positively charged. It is easy for the increase of molecular weight and volume, which results in the enhancement of scattering intensity. In addition, the hydrophobic force was contributed to the enhancement of the scattering intensity. All of results showed that the increments of resonance Rayleigh scattering intensity were linear to the concentrations of dermatan sulfate in certain ranges.

\section{ACKNOWLEDGEMENTS}

This project is supported by National Youth Science Fund of China No. 21202110.

\section{REFERENCES}

1. T.M. Zhang and W.T. Wu, Animal Biochemical Pharmaceutics, People's Medical Publishing House, Beijing, pp. 210-215 (1981)

2. F.A. Ofosu, M.R. Buchanan, N. Anvari, L.M. Smith and M.A. Blajchman, Ann. N. Y. Acad. Sci., 556(1 Heparin and R), 123 (1989).

3. T. Oguma, S. Tomatsu, A.M. Montano and O. Okazaki, Anal. Biochem., 368, 79 (2007).

4. L. Jaques, S.Wice and L. Hiebert, J. Lab. Clin. Med. 115, 422 (1990).

5. X.H. Wang, J.D. Yang and J.T. Liu, Chem. Reagents, 30, 853 (2008).

6. X.L. Hao, N.B. Li and H.Q. Luo, Spectrochim. Acta A, 71, 1673 (2009).

8. X.Q. Wei, Z.F. Liu and S.P. Liu, Anal. Biochem., 346, 330 (2005).

9. D.W. Deng, J.S. Yu and Y. Pan, J. Colloid Interf. Sci., 299, 225 (2006).

10. S.P. Liu, Z.L. Jiang, L. Kong and Q. Liu, Sci. China Series B, 45, 616 (2002). 